\title{
After 62 years of regulating immunity, dexamethasone meets COVID-19
}

Derek W. Cain ${ }^{1}$ and John A. Cidlowski ${ }^{凶}$

Six months into the COVID-19 pandemic, effective treatments have been frustratingly sparse.

The RECOVERY clinical trial, however, revealed that treatment with dexamethasone, a classic

synthetic glucocorticoid, enhanced survival of critically ill patients with COVID-19. In this

Comment, we discuss the immunological impacts of glucocorticoid therapy for COVID-19.

A frustrating challenge in the ongoing coronavirus disease 2019 (COVID-19) pandemic has been the lack of effective treatments. The antiviral drug remdesivir, for example, was found to reduce the time to recovery of patients hospitalized with COVID-19, but it had only a marginal effect on mortality ${ }^{1}$. Thus, considerable excitement surrounded a press release on 16 June 2020 (and subsequent report ${ }^{2}$ ) by researchers from the RECOVERY (Randomized evaluation of COVID-19 therapy) clinical trial announcing the synthetic glucocorticoid dexamethasone as a potential treatment. Not only does dexamethasone reduce COVID-19-related mortality, but it is cheap, widely available and comes with 60 years of safety profiling - bright news in a dreary season of world events.

Glucocorticoids have been investigated before in animal models and small clinical trials for infections with different coronaviruses, but the results were mixed. In one of the earliest publications of the 2003 severe acute respiratory syndrome coronavirus (SARS-CoV) outbreak, glucocorticoid treatment was noted to offer some therapeutic benefit ${ }^{3}$, whereas a more recent meta-analysis of glucocorticoid therapy for SARS-CoV, Middle East respiratory syndrome coronavirus (MERS-CoV) and SARS-CoV-2 raised a cautionary flag, citing delayed viral clearance and a lack of evidence for improved patient outcomes ${ }^{4}$. However, the RECOVERY report was deemed suitably robust that it has already changed the standard of care for COVID-19 treatment. The key finding of this trial centres on the condition of the patient with COVID-19 at initiation of dexamethasone therapy: mortality of patients on ventilators dropped by one-third, and mortality of patients on oxygen support without ventilation reduced by one-fifth, whereas patients not receiving respiratory support did not see any benefit. In this Comment, we seek to arm the immunologist with a basic understanding of glucocorticoid biology as a framework for thinking about dexamethasone treatment for COVID-19. We stress that, for dexamethasone treatment of COVID-19, timing and dose are everything.
Dexamethasone, along with naturally occurring cortisol and its synthetic counterparts, binds to intracellular glucocorticoid receptors (GRs), which are ligand-dependent transcription factors expressed by most cells of the body. Liganded GRs translocate from the cytosol to the nucleus to modulate a host of cellular processes. Genomic effects occur through direct binding to glucocorticoid response elements in target genes, thereby positively or negatively regulating transcription. Liganded GRs also bind to other transcription factors via protein-protein interactions, enhancing or inhibiting their capacity to affect gene transcription. GRs can also rapidly affect non-genomic cell processes through interactions with plasma membranes, interference with cytoplasmic signalling cascades and mitochondrial translocation. Thus, in contrast to many biologicals and small-molecule inhibitors with very specific molecular targets, glucocorticoids cause widespread changes in cellular transcriptomes and processes.

Although glucocorticoids impact many physiological pathways, including metabolism, cell growth and apoptosis, the benefits of dexamethasone for patients with COVID-19 likely arise from its immunosuppressive properties. Many immunomodulating effects of glucocorticoids reflect cell type-specific changes in the transcriptome $e^{5}$, tempering the specialized activities of different immune cell types. In B cells, for example, glucocorticoids downregulate genes associated with B cell receptor and Toll-like receptor 7 signalling, while upregulating the anti-inflammatory cytokine IL-10 (REF.5.). Similarly, glucocorticoids act on T cells to dampen $\mathrm{T}$ cell receptor signalling and cytokine expression. In dendritic cells, glucocorticoids inhibit antigen presentation, expression of co-stimulatory molecules and cytokine production. Indeed, some of the most potent anti-inflammatory actions of glucocorticoids occur by inhibiting the production of pro-inflammatory cytokines (IL-1, TNF and IL-6), chemokines and other soluble mediators (such as prostaglandin $\mathrm{E}_{2}$, leukotrienes and histamine) that act on the vasculature to promote vasodilation and leukocyte recruitment. Although many 
of these effects occur through glucocorticoid-mediated alterations in gene transcription, inhibitory interactions between GRs and the transcription factors $\mathrm{NF}-\kappa \mathrm{B}$ and AP-1 are important modes of glucocorticoid anti-inflammatory action, as these transcription factors serve as 'hubs' at the termini of several signalling pathways, including those downstream of Toll-like receptors, cytokine receptors, $\mathrm{Fc}$ receptors, and $\mathrm{B}$ and $\mathrm{T}$ cell receptors ${ }^{6}$.

An important observation from the RECOVERY trial was that dexamethasone provided benefit only to severely ill patients with COVID-19. To understand why dexamethasone treatment may be restricted in utility to the most critical cases, we can overlay glucocorticoid effects on the progression from initial infection to severe disease ${ }^{7}$. Infection begins with SARS-CoV-2 binding to angiotensin-converting enzyme 2 (ACE2), which is expressed on cells in various tissues and organs but is particularly high on type 2 pneumocytes in the lung. During the early incubation stage, subjects are generally asymptomatic and viral replication is inhibited by the type I interferon response. With successful replication and spreading of SARS-CoV-2, the disease progresses with mild to moderate symptoms (including sore throat, cough, fever and muscle aches) that arise from both virus-associated tissue damage in the respiratory tract and antiviral activity of the adaptive immune system, of which interferon- $\gamma$-secreting $\mathrm{CD} 4^{+} \mathrm{T}$ helper 1 cells and $\mathrm{CD}^{+}$cytotoxic $\mathrm{T}$ cells seem to be paramount. In most infected people, mild or moderate symptomatic disease is successfully resolved through a coordinated antiviral immune response. However, in some people - for reasons that are not yet clear - the immune response goes awry at this point. We suspect that the amount of cell death and tissue damage, due to virus replication, immune system activity and effects of any additional co-morbidities, reaches a threshold at which so-called alarmins or damage-associated molecular patterns (DAMPs) come into play. Alarmins describe the diverse set of endogenous molecules (such as heat shock proteins, ATP, uric acid, HMGB1, IL-1a and IL-33) that are released upon unregulated cell death and/or degranulation ${ }^{8}$. The release of alarmins, on top of unresolved viral infection, results in a hyperinflammatory state characterized by systemic increases in cytokines and chemokines ('cytokine storm'), which triggers neutrophil and monocyte recruitment, vascular leakage and further tissue damage. Pathology at this stage likely reflects an uncontrolled, feedforward loop in which inflammation causes tissue damage that triggers more inflammation, and so on. If it is not brought under control, this hyperinflammatory state manifests as acute respiratory distress syndrome, sepsis and, eventually, organ failure.

There is a growing movement to approach COVID-19 as a two-phase disease: in the early phase, virus pathology dominates; and in the later phase, immunopathology drives disease. In thinking about COVID-19 this way, perhaps it is not surprising that dexamethasone offered no benefit to patients in the RECOVERY trial whose disease had not progressed to a stage necessitating respiratory support; indeed, the immunosuppressive effects of glucocorticoids at this stage of disease might hamper antiviral responses. It is in the later, hyperinflammatory phase of COVID-19 that the immunomodulatory effects of glucocorticoids are beneficial, perhaps by breaking the inflammatory feedforward loop, at least in some patients.

As a closing point, we highlight the dose of dexamethasone used in the RECOVERY trial: $6 \mathrm{mg}$, once a day. Although this feature of the study did not make the headlines, there are important implications to this number. Glucocorticoids elicit dose-specific responses owing to differential expression of GRs and transcriptional cofactors across different cell types, alongside cell type-specific chromatin accessibility landscapes?. A $6 \mathrm{mg}$ per day dexamethasone regimen is a fairly low-dose therapy - for comparison, doses for treatment of shock, multiple sclerosis and multiple myeloma are $20-40 \mathrm{mg}$ per day. At $6 \mathrm{mg}$ per day, patients suffer minimal side effects such as skin thinning, hypertension, weight gain, osteoporosis and diabetes. Moreover, at this low dose, there is likely a much reduced incidence of glucocorticoid resistance, a condition in which glucocorticoid administration renders the subject insensitive to further glucocorticoid treatment ${ }^{10}$. We suspect that low-dose dexamethasone treatment complements endogenous cortisol activity to suppress COVID-19associated immunopathology, while avoiding the adverse effects of high-dose glucocorticoid therapy.

We suggest that differences in timing and dose underlie many of the inconsistent, and sometimes conflicting, results across studies of glucocorticoid therapy. With a little more attention to these numbers, dexamethasone and other synthetic glucocorticoids will likely continue to be a potent option for treating diseases with immunopathological aetiologies.

1. Beigel, J. H. et al. Remdesivir for the treatment of COVID-19preliminary report. N. Engl. J. Med. https://doi.org/10.1056/ NEJMoa2007764 (2020).

2. Horby, P. et al. Dexamethasone in hospitalized patients with Covid-19 - preliminary report. N. Engl. J. Med. https://doi.org/ 10.1056/NEJMoa2021436 (2020).

3. Lee, N. et al. A major outbreak of severe acute respiratory syndrome in Hong Kong. N. Engl. J. Med. 348, 1986-1994 (2003).

4. Li, H. et al. Impact of corticosteroid therapy on outcomes of persons with SARS-CoV-2, SARS-CoV, or MERS-CoV infection: a systematic review and meta-analysis. Leukemia 34, 1503-1511 (2020).

5. Franco, L. M. et al. Immune regulation by glucocorticoids can be linked to cell type-dependent transcriptional responses. J. Exp. Med. 216, 384-406 (2019)

6. Cain, D. W. \& Cidlowski, J. A. Immune regulation by glucocorticoids. Nat. Rev. Immunol. 17, 233-247 (2017).

7. Oberfeld, B. et al. SnapShot: COVID-19. Cell 181, 954 (2020).

8. Yang, D., Han, Z. \& Oppenheim, J. J. Alarmins and immunity. Immunol. Rev. 280, 41-56 (2017).

9. Reddy, T. E., Gertz, J., Crawford, G. E., Garabedian, M. J. \& Myers, R. M. The hypersensitive glucocorticoid response specifically regulates period 1 and expression of circadian genes. Mol. Cell. Biol. 32, 3756-3767 (2012).

10. Ramamoorthy, S. \& Cidlowski, J. A. Ligand-induced repression of the glucocorticoid receptor gene is mediated by an NCoR 1 repression complex formed by long-range chromatin interactions with intragenic glucocorticoid response elements. Mol. Cell. Biol. 33, 1711-1722 (2013).

Acknowledgements

Research from our laboratory has been supported by grant 1ZIA ES090079 24 from the NIEHS/NIH.

Competing interests

The authors declare no competing interests. 